\title{
Les occupants de bâtiments tertiaires performants en énergie : entre logiques d'usage, salariale et domestique
}

\author{
Isabelle Garabuau-Moussaoui ${ }^{1, \mathrm{a}}$ avec la participation de Sarah Thiriot ${ }^{2}$ \\ ${ }^{1}$ EDF R\&D, 1 avenue du Général de Gaulle, 92141 Clamart Cedex, France \\ ${ }^{2}$ UMR Pacte, Institut d'études politiques, BP. 48, 38040 Grenoble Cedex 9, France
}

\begin{abstract}
Résumé. L'occupant de bâtiment performant en énergie se trouve au croisement de trois logiques d'occupation : la logique d'usage, co-construite par l'usager et les concepteurs de ce type de bâtiment ; la logique salariale, co-construite par le salarié et la direction occupante ; et la logique citoyenne, co-construite par l'individu dans la sphère domestique et par les politiques publiques. Ces trois logiques s'entrechoquent autour de dispositifs de tension : l'aménagement des espaces et en particulier l'open space, les définitions divergentes de l'environnement et la notion de citoyenneté. Derrière ces logiques et ces dispositifs de tension, les deux enjeux des occupants, usagers-salariés-membres d'un ménage, sont le confort et le juste partage des responsabilités, dans cet espace social qu'est l'entreprise.
\end{abstract}

\begin{abstract}
Several qualitative studies in labeled energy-efficient buildings of the tertiary sector (companies, schools) show that the occupants of this kind of buildings are located at the crossroads of three social logics: the logic of use, that is co-constructed by users and buildings' designers; the professional logic, co-constructed by the employees and the occupying company; and the domestic logic, co-constructed by the individuals as household's members and by the public policies. These three logics collide and create tensioning devices: 1) spatial planning and in particular the question of open plan offices, 2) divergent definitions of the environment and 3) questions concerning citizenship. Behind these logics and these tensions, the two issues for the occupants (as users, employees, members of a household) are comfort and the fair sharing of responsibilities in this social space that is the company.
\end{abstract}

\section{Introduction}

L'analyse des politiques d'entreprises en matière d'énergie demande à déconstruire les termes utilisés pour appréhender cette nouvelle réalité. Bien entendu, on peut questionner la notion de "politiques », en cherchant par exemple le contexte politique structurant les nouvelles problématiques énergétiques [1] ou en étudiant les processus de décisions des entreprises, entre contraintes réglementaires et actions volontaires, pour comprendre leurs enjeux à s'engager dans l'efficacité énergétique [2, 3].

\footnotetext{
ae-mail : isabelle.moussaoui@edf .fr
}

This is an Open Access article distributed under the terms of the Creative Commons Attribution License 4.0, which permits unrestricted use, distribution, and reproduction in any medium, provided the original work is properly cited. 
On peut également questionner les termes d'énergie, de performance, d'efficacité énergétique, entre affichage de principes, de standards, d'objectifs, d'une part, et économies observées, réalisées, d'autre part [4].

Mais on peut également interroger la notion même d'entreprise dans ce cadre. En effet, il s'avère que ce processus de décision de la performance énergétique, dans un contexte de « pression » à agir en matière de politique publique de l'énergie, renvoie à une constellation d'acteurs très différents, créant un système d'action concret, dans et hors de l'entreprise, qui participent de la construction d'un dispositif sociotechnique complexe, le «bâtiment performant en énergie » [5]. Ce système d'action peut être « déroulé » historiquement (par phases de décision) et par structures organisationnelles (les différentes parties prenantes et leurs relations). L'entreprise n'est donc pas une entité abstraite, uniforme (elle est composée d'acteurs décisionnaires variés) et n'est pas isolée (elle est encastrée dans des réseaux, des systèmes d'action, une dynamique, etc.).

Interroger la notion d'entreprise permet de comprendre que le processus de décision en matière de performance énergétique est un cheminement long, empli de compromis, voire de conflits, de stratégies et d'enjeux mais aussi de croyances, de contraintes, d'influences multiples portés par des acteurs aux rationalités multiformes [6].

Dans ce cadre, une figure composite apparaît comme dépositaire d'un ensemble de logiques, de rationalités et de processus de décision : l'occupant du bâtiment performant en énergie. Au moins trois logiques, co-construites par les différents acteurs en présence dans les projets, se combinent dans la notion « d'occupant» :

- la logique d'usage du bâtiment, qui se crée sur une tension entre les actions de conception, construction, maintenance, exploitation et utilisation des bâtiments performants en énergie, et qui se matérialise dans un «script technologique »;

- la figure salariale, qui est traversée dans les projets de rénovation/construction par la question du déménagement et du rapport à la « Direction », cette dernière utilisant plus ou moins les bâtiments comme « vitrine » à l'extérieur et comme outil de management/conduite du changement à l'intérieur ; et les salariés cristallisant dans le bâtiment des problématiques de rapport à la culture d'entreprise et aux modes de management et de construction de la confiance/défiance envers leur entreprise ;

- la figure domestique renvoyant aux tensions entre des valeurs personnelles et des contraintes de situation dans différentes arènes de vie.

Ces trois figures, renvoyant chacune à des tensions de gestion de l'énergie, se déploient autour de trois dispositifs intermédiaires de tension : l'aménagement des espaces (open space, par exemple), la définition de l'environnement et la posture « citoyenne ».

Ces trois «problématiques » traversent en effet les actions concrètes des différents acteurs rencontrés dans les projets de construction/rénovation, et c'est à un compromis différent et unique que l'on assiste à chaque projet de bâtiment performant.

\section{Méthode}

Cet article s'appuie sur une série d'enquêtes réalisées par et/ou pour EDF R\&D, certaines en partenariat. En particulier, les exemples seront tirés de :

- l'enquête réalisée conjointement avec le CREDOC en 2011, dans deux entreprises de bureaux implantées en Île-de-France, appelées ici Le Labo et Consulting [1] ;

- une enquête réalisée en 2011-2012 dans un bâtiment de bureaux avec rénovation lourde en région Rhône-Alpes (entreprise appelée ici L'Ingénierie) ; 
- une enquête réalisée en 2013 par Sarah Thiriot, dans le cadre d'un stage de Master 2 à l'IEP de Grenoble, en partenariat entre PACTE et EDF R\&D, piloté par Thomas Reverdy et Isabelle Moussaoui, dans un bâtiment de bureaux : La Plateforme en PACA [7] ;

- une enquête réalisée en 2012 dans une école basse consommation (L’École).

La plupart des enquêtes ont donc été réalisées dans des entreprises de bureaux (4 bâtiments). Nous y adjoignons des exemples et des analyses provenant de l'enquête sur une école, même si l'école n'est pas une entreprise. En effet, de nombreux mécanismes sociaux de conception, d'exploitation et d'utilisation sont communs et peuvent être généralisés sous le vocable « secteur tertiaire » (comprenant des bâtiments publics et privés).

Si les dispositifs d'enquête ne sont pas tout à fait équivalents sur les différents terrains, la démarche générale est commune : comprendre les pratiques énergétiques des occupants, en pensant le bâtiment comme un dispositif sociotechnique, c'est-à-dire conçu, mis en œuvre et utilisé « socialement », par des acteurs, dans des relations sociales, des rapports sociaux, des systèmes culturels, de croyance, de pratiques. Pour cela, dans la mesure du possible pour chaque terrain, l'enquête a porté non seulement sur les occupants et leurs usages, mais également sur la chaîne d'acteurs ayant conçu le bâtiment (ou porté sa rénovation) et sur les acteurs parties prenantes de son exploitation.

L'analyse transverse réalisée ici tire des éléments des analyses réalisées dans le cadre de ces différentes enquêtes, mais développe également de nouveaux aspects non mis en perspective jusque-là.

\section{Les trois logiques de l'occupant}

Les différentes enquêtes réalisées montrent que se développent des figures de l'occupant du bâtiment performant en énergie, au croisement de trois logiques d'action, l'occupant étant à la fois défini comme usager, salarié et membre d'un ménage.

\subsection{La logique d'usage (usager/bâtiment)}

Il pourrait paraître évident que pour les concepteurs des bâtiments performants en énergie, l'occupant soit tout d'abord un usager. Cela est vrai dans le sens de la focalisation sur le bâtiment, ses systèmes et ses usages (dont la consommation d'énergie), mais cela est à relativiser fortement si l'on considère la notion d'acteur du bâtiment et des systèmes.

En effet, les concepteurs de bâtiments et de systèmes techniques construisent une figure de l'occupant comme « un acteur dans un script technologique » [8-10], c'est-à-dire qu'ils souhaitent que l'occupant ait peu de marges de manœuvre face aux systèmes.

Dans certains cas, les concepteurs « optent » (sous contrainte, voir [6]) pour des systèmes très automatisés. Ces derniers sont censés être «transparents » pour les occupants, dont les comportements sont considérés comme "pas raisonnables », " anarchiques », voire " dangereux ${ }^{\mathrm{b}}$ " pour le fonctionnement des systèmes et la performance énergétique. Leur conception est que la maîtrise des consommations énergétiques, enjeu de certification $\mathrm{HQE} / \mathrm{BBC}$, n'est possible que si les occupants ne s'en mêlent pas, car ils sont facteurs d'incertitude. Ce sont les systèmes qui doivent « gérer » les consommations énergétiques et «piloter » les usages, dans une logique très technicienne et « experte » (maîtrise de la technique et technologisation de la maîtrise).

Dans d'autres cas, l'occupant est censé avoir un rôle à jouer dans l'usage du bâtiment et de ses systèmes, dans une conception semi-automatique. Mais alors, l'occupant serait à « éduquer » par

${ }^{\mathrm{b}}$ Extraits d'entretiens des enquêtes sur Le Labo, L'Ingénierie et La Plateforme. 


\section{SHS Web of Conferences}
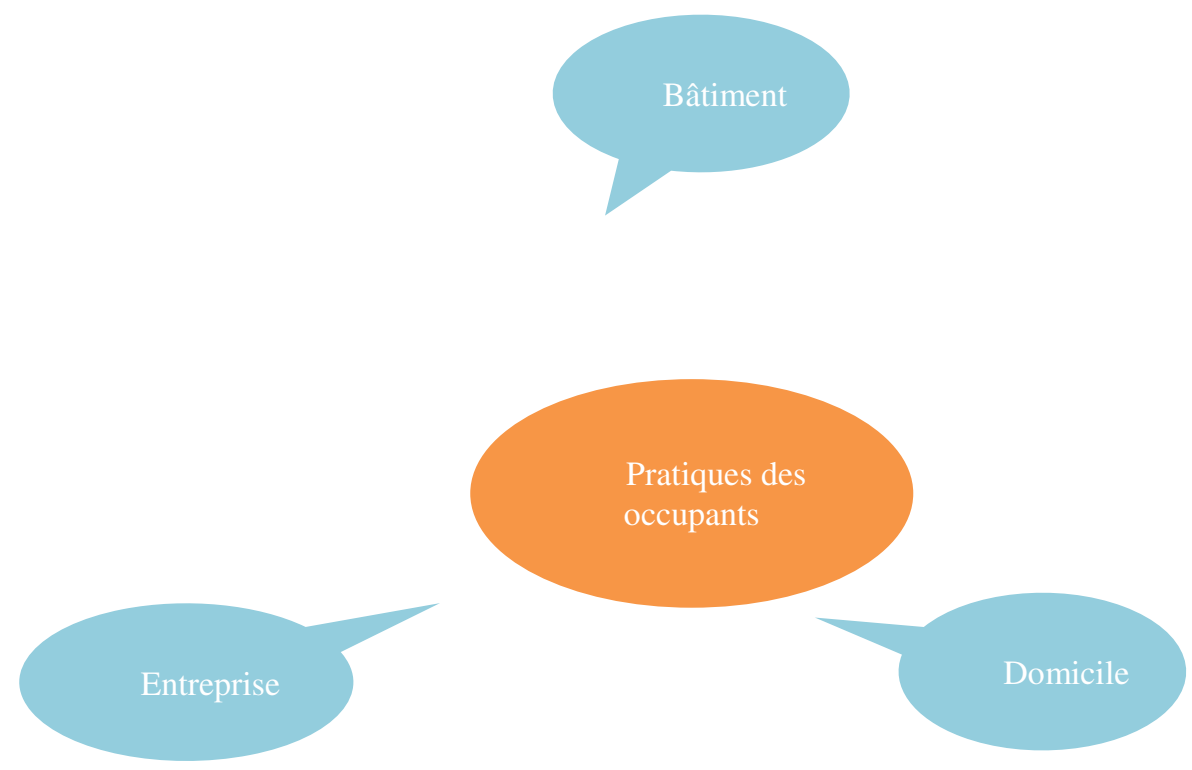

Figure 1. Les trois logiques de rapport à l'énergie des occupants.

le bâtiment et ses systèmes, ou par les gestionnaires du bâtiment, ou encore par la hiérarchie des salariés, donc serait "ignorant », voire même mal éduqué. Le centrage est donc toujours du côté de la technique, des systèmes, d'une part, et des experts d'autre part, qui peuvent apporter une " pédagogie environnementale » aux usagers ${ }^{\mathrm{c}}$.

Dans les deux cas, l'usager est a priori perturbateur et doit s'adapter aux systèmes, aux consignes, aux objectifs de performance, soit par la non-action, soit par une action « correcte » sur les systèmes (c'est-à-dire une action inscrite dans le script technologique), soit par un effort d'apprentissage, fait de pratiques cadrées et de valeurs transmises par le bâtiment, ses systèmes, et ses acteurs d'exploitation.

Les occupants, quant à eux, ont une appréhension du bâtiment et de ses systèmes bien différente. Ils «pratiquent » et s'approprient le bâtiment en tant que lieu de vie et de travail, et non pas en tant que dépositaire d'un objectif de performance énergétique. Ils utilisent les systèmes dans une logique de confort et de conditions de travail. Les systèmes ne sont pas vécus comme transparents, d'une part parce que les occupants souhaitent s'en emparer, s'en saisir, dans une logique d'appropriation, et d'autre part, parce que ces systèmes connaissent des dysfonctionnements, aux dires des occupants. Il existe un écart fort entre des systèmes poussant à la standardisation et à la moyennisation des comportements versus des occupants poussant à l'appropriation, voire la personnalisation des espaces et des équipements. Pour les occupants, le bâtiment n'est pas un donné, il est un construit, qui doit devenir un « lieu de travail ». Ce lieu de travail peut être approprié de plusieurs manières, par exemple dans une logique «familiale/domestique », comme deuxième chez-soi, "cocon », " grotte de hobbitt » (Consulting), soit dans une logique plus « industrielle », de performance et d'équipements « modernes » (Le Labo, L'Ingénierie). Dans tous les cas, le lieu de travail doit s'adapter aux activités professionnelles et les occupants développent alors une logique salariale.

\footnotetext{
c C'est le cas à L'École, dont les parties prenantes proclament l'objectif pédagogique de l'établissement, et à Consulting, dont le directeur est un «militant technophile» (voir [1]).
} 


\subsection{La logique salariale (entreprise/salariés)}

La logique salariale est très importante car les objectifs principaux des occupants se situent dans un registre organisationnel et non pas dans un registre architectural ou énergétique.

Dans cette logique, le binôme central n'est donc plus concepteurs/occupants, mais direction/salariés. Chacun doit intégrer la conception du bâtiment dans ses actions et ses relations, mais cela crée des tensions.

Tout d'abord, l'arrivée dans un bâtiment performant suppose un projet de déménagement (dans les cas étudiés, même en cas de rénovation, les salariés ne travaillaient pas dans le bâtiment avant rénovation). Ce projet structure fortement la manière dont les occupants vont " percevoir », construire une vision du monde autour du bâtiment. En effet, si le déménagement est tendu socialement, ou si les occupants considèrent «perdre » un certain nombre d'avantages dans le déménagement, l'entrée dans le bâtiment sera plus difficile et le bâtiment sera plus critiqué que lorsque les salariés ont l'impression, individuellement ou collectivement, de gagner avec le déménagement. Les critères de jugement sont assez variés : temps de transport, emplacement par rapport aux lieux de vie urbains, conditions matérielles de travail et équipement, surface par personne, types de bureaux, réorganisation des services, etc. Ces critères renvoient à la fois à l'inscription du lieu de travail dans la ville, au rapport à la direction et à la manière dont elle accompagne le déménagement, au contexte économique global, ou encore au confort.

L'entrée dans le bâtiment est en particulier un analyseur et un catalyseur des rapports sociaux dans l'entreprise. La confiance dans l'employeur, la légitimité de celui-ci à proposer un nouvel espace de travail vont influencer la manière de percevoir le bâtiment.

Par exemple, la plupart des bâtiments étudiés sont des bâtiments « vitrine » pour les entreprises qui s'y installent (au niveau national des entreprises, tout au moins). Les discours des employés sur les inaugurations sont assez caractéristiques d'une confiance ou d'une méfiance, et de relations proches ou éloignées entre directions et salariés. Dans toutes les enquêtes réalisées, les inaugurations officielles, auxquelles les salariés ne sont pas invités, sont perçues de manière assez critique par les salariés. Dans les entreprises où une inauguration «interne » a eu lieu, elle a été très appréciée, et dans les entreprises où elles n'ont pas eu lieu, les salariés l'ont déploré. Selon eux, cette absence d'inauguration symbolise une distance de leur employeur vis-à-vis d'eux en tant qu'entité organisationnelle et géographique : » Nous avions un directeur qui nous avait dit, «on va faire un truc» et puis entre-temps, il est parti, voilà et puis cela tombe aux oubliettes... »(La Plateforme).

Notons que les directions sont également tiraillées entre le caractère performant et « vitrine » des bâtiments et la gestion de leurs équipes, surtout dans les cas où le déménagement crée des tensions entre partenaires sociaux. Par exemple, à L'Ingénierie, la direction locale devait gérer à la fois la volonté de la direction nationale de valoriser le label HQE et le caractère innovant du bâtiment et la crainte que ces éléments ne viennent envenimer des négociations sociales sur les nouvelles conditions de travail dans le bâtiment. La réunion d'information et de sensibilisation aux économies d'énergie a ainsi été sans cesse reportée, aucun acteur partie prenante de la rénovation ne souhaitant l'organiser, arguant qu'elle était très importante, mais pas dans ses attributions. La direction locale a donc été « contrainte »d'organiser cette réunion, avec un discours très ambivalent auprès des salariés, les enjoignant d'être acteurs tout en respectant les consignes; et mettant en avant le caractère performant en énergie du bâtiment, tout en cherchant à minimiser les effets que cela pourrait avoir sur les conditions de travail.

\subsection{La logique « domestique »}

L'énergie est de prime abord, pour les individus, une thématique domestique. Bien que la consommation d'énergie ne soit pas une pratique en tant que telle, mais un moyen de réaliser des activités, l'attention 
énergétique, les économies d'énergie sont devenues une problématique au croisement des politiques publiques et des comportements privés [11-14]. C'est beaucoup plus récemment que la problématique se pose dans les entreprises, en lien notamment avec le développement des labels et certifications environnementales et énergétiques (ISO 14 001, HQE, BBC).

Cela crée des repositionnements concernant les frontières entre le privé et le professionnel. L'attention énergétique s'est construite dans le domaine privé, dans la socialisation, les expériences et les apprentissages des individus, en tant que membres d'un ménage ${ }^{\mathrm{d}}$, et est nouvellement investie par les directions et par les concepteurs de bâtiments. Les occupants interrogent donc la légitimité de ces deux acteurs à leur prescrire ou interdire des comportements considérés comme " hors travail ». Cette interrogation, si elle est nouvelle concernant l'énergie, fait suite à des hybridations de même type concernant d'autres activités comme l'alimentation [15], l'hygiène ou les nouvelles technologies [16]. De nombreuses activités sur le lieu de travail sont dans un entre-deux entre domaine discrétionnaire et contrainte, entre comportements privés et comportements professionnels. L'énergie participe de ces activités. Les salariés construisent donc des catégories de jugement des demandes de l'employeur, entre « légitime» et «illégitime »; et des catégories de pratiques, entre «négociables » et «non négociables », certaines relevant plus de leurs comportements personnels, c'est-à-dire d'une logique domestique que de la logique salariale.

Les entreprises occupant des bâtiments performants sont poussées par les acteurs du bâtiment (architectes, bureaux d'études, direction immobilière, exploitant, facility manager) à inciter leurs salariés à intégrer l'attention énergétique dans leurs pratiques, mais en même temps sont rétives à aller sur le terrain personnel et à mettre des objectifs non professionnels supplémentaires à leurs salariés. Une partie des salariés réagit d'ailleurs sur la contradiction des objectifs : "S'ils veulent vraiment que je fasse des économies d'énergie, je peux éteindre mon ordinateur et rentrer chez moi » est une phrase entendue plusieurs fois pendant les enquêtes.

\section{Les dispositifs intermédiaires de tension}

Si des conflits et des tensions apparaissent au cœur même de chaque logique (usage salarial et domestique), l'analyse montre que certaines questions viennent concentrer les tensions, en particulier parce qu'elles font s'entrechoquer ces différentes logiques. Dans nos enquêtes, nous avons trouvé trois problématiques de ce type : l'aménagement des bureaux, les définitions de l'environnement et la question de la citoyenneté.

\subsection{Entre performance professionnelle et énergétique : l'espace de travail et l'open space}

L'open space est un agencement de l'espace prôné dans les réalisations de bâtiments performants en énergie, en raison de la simplification de la gestion des flux qu'il implique. De plus, les directions des entreprises qui occupent les locaux y voient une manière de réduire le nombre de mètres carrés par personne, pour réduire les coûts d'investissement et de fonctionnement. Les deux logiques (bâtiment et managériale) se combinent en faveur des plateaux ouverts. Cependant, cet agencement est plus ou moins bien vécu par les occupants.

L'appréciation de l'open space dépend tout d'abord des conditions antérieures de travail. Si les occupants étaient auparavant en bureaux fermés, l'open space est mal vécu, considéré comme une régression des conditions de travail (ex. à L'Ingénierie, où les salariés ont refusé la disposition en plateaux ouverts). Par contre, si les usagers étaient déjà en open space précédemment, ils peuvent

\footnotetext{
d Et éventuellement en tant que membre d'un collectif citoyen (communautés énergétiques, partis politiques, associations, etc.).
} 
être davantage sensibles aux améliorations apportées par le nouvel agencement du lieu de travail. Ainsi, dans le cas de La Plateforme, le bâtiment précédent était vétuste et le nouveau est apprécié : selon les salariés, la lumière naturelle, l'aménagement neuf, moderne et "très ouvert » font du lieu de travail « un bâtiment de notre époque », ce qui « englobe plein de choses, la démarche écolo, la performance thermique... Enfin c'est un ensemble »; "Par rapport à [l'ancien bâtiment], on a changé de millénaire ». Le bâtiment est qualifié d' "agréable ».

De plus, l'appréciation de l'open space dépend de l'activité professionnelle. Si l'open space est « imposé » en dehors de l'activité, il est rejeté. Ainsi, dans une entreprise où un étage a été rénové en open space, l'équipe est mécontente car ses membres travaillent sur plans de grandes dimensions et ne peuvent plus les attacher aux murs (puisqu'il n'y a plus de murs). Tout un ensemble de tactiques et de détournements sont mis en place pour pallier le manque de supports verticaux.

En revanche, si l'open space est pensé en cohérence avec l'activité, il est apprécié. Ainsi, à $L a$ Plateforme, deux groupes professionnels distincts se partagent les locaux : une équipe de conseillers clientèle, qui apprécient l'open space, parce qu'il permet les coopérations et la mutualisation des connaissances, que le bâtiment a un bon confort acoustique, et parce qu'ils ont l'habitude de cet agencement de travail (front office et back office possibles sur le même espace de travail, contrairement à l'ancien bâtiment où ils devaient se déplacer pour les appels téléphoniques). L'autre groupe professionnel, que l'on peut qualifier «d'experts autonomes », apprécie les bureaux fermés du $1^{\text {er }}$ étage, leur permettant une gestion personnalisée de leur activité, de leurs déplacements (ils sont «nomades ») et de leur rythme de travail (ils peuvent avoir des horaires décalés). Le bâtiment correspond, dans ce cas, à la spatialisation des activités professionnelles.

L'appréciation de l'aménagement de l'espace de travail dépend également de la culture d'entreprise, des modes de coopération et de la génération de ses occupants. Ainsi, à Consulting, petite entreprise de conseil, où les salariés sont jeunes, l'open space est considéré comme normal, permettant une sociabilité et des rapports de travail fluides. Il entre en cohérence avec un mode d'appropriation du lieu de travail, basé sur la convivialité et le collectif. Cependant, les plus anciens ressentent une certaine « fatigue » de l' open space, obligeant à des tactiques de repli sur soi (casque sur les oreilles).

La question de la transparence traverse les projets architecturaux de bâtiments performants, que ce soit concernant l'open space ou les locaux vitrés. Si, pour les concepteurs de bâtiments cela apporte des solutions en termes de flux (lumière, air, etc.), pour le management et pour les salariés cela renvoie à la gestion de binômes tels que privé/professionnel, contrôle/liberté, personnalisation/standardisation [17]. L'espace se construit en territoire pour les salariés, qui y investissent matériellement, socialement et symboliquement. «Derrière » des objectifs de performance énergétique, se jouent des rapports sociaux entre individus, entre groupes professionnels ou entre institutions. Par exemple, à L'École, les salles de classe sont investies par les enseignants dans leur pédagogie, par l'affichage de consignes, règles, fresques, dessins d'enfants, etc. La nouvelle école bioclimatique est construite sur le principe de la luminosité naturelle, et possède donc de grandes baies vitrées sur deux murs de chaque classe (une vitre donnant sur l'extérieur et une vitre donnant sur le couloir pour que celui-ci bénéficie de la lumière naturelle). Cette configuration est vivement critiquée et rejetée par les enseignants, qui pensent que cela les empêche de réaliser leur travail correctement, et qui continuent donc d'afficher sur les vitres. Une enseignante, très sensibilisée à l'environnement et ayant intégré le discours et les objectifs architecturaux de l'école, écrit avec un feutre spécial sur les vitres, ce qui permet de limiter la perte d'apport lumineux. Cette solution n'est pas reprise par ses collègues car la raison de leur « résistance » est en fait ailleurs : dans le refus que la municipalité (propriétaire du bâtiment) ne vienne s'immiscer dans leurs pratiques pédagogiques (par le cadrage des pratiques que le bâtiment impose). Les vitrages et la gestion des espaces cristallisent un rapport aux acteurs intervenant dans l'école.

Cela peut être généralisé aux autres enquêtes : l'open space et la gestion des espaces cristallisent le rapport entre salariés et direction dans un contexte de déménagement. Si ce dernier est perçu positivement par les salariés (amélioration des conditions de travail, de la gestion des équipes, des 
conditions de transport, de l'environnement du lieu de travail), ils appréhenderont les agencements spatiaux et plus globalement le nouveau bâtiment plus positivement que si le déménagement se passe dans des rapports tendus avec la direction (suspicion de réaliser des baisses d'effectifs par le déménagement, difficultés d'accès ou augmentation du temps de transport, redispatchage des équipes et des bureaux en défaveur des salariés, etc.).

Ensuite, dans l'usage quotidien du lieu de travail, les salariés vont être en lien avec les systèmes, les automatismes, les cadrages de pratique du bâtiment performant en énergie. L'open space est le symbole et la matérialisation d'une gestion collective et peu personnalisée du confort (discours d'homogénéité), en même temps qu'il peut induire de vraies inégalités dans la répartition des contraintes (les salariés sous les bouches d'aération se plaignent de l'effet « douche »; l'orientation sud/nord induit des différences de luminosité et de chaleur importantes, etc.) et laisse généralement peu de marges de manœuvre pour agir sur les systèmes (gestionnaires d'énergie qui ne sont pas laissés à la main des occupants, ou pilotant de très grandes zones).

Au contraire, le bureau fermé est le symbole et la matérialisation de la personnalisation, de l'appropriation de l'espace. Mais les salariés bénéficiant d'un bureau fermé ne sont pas maîtres de leurs décisions dans un bâtiment performant pour autant. Les automatismes, les dispositifs de gestion de l'énergie, viennent fortement cadrer les pratiques. Ainsi, à L'Ingénierie, la gestion des flux « demande » à ce que les portes soient fermées le jour (maintenir la chaleur ou la fraîcheur dans le bureau) et ouvertes la nuit (pour que la ventilation renouvelle l'air), alors que la culture de cette entreprise développe les pratiques contraires : laisser les portes ouvertes la journée, pour avoir des relations fluides avec les collègues qui «passent », et fermer la porte la nuit, parce que le bureau est considéré comme un espace personnel, voire parfois comme contenant des données sensibles. La porte, les passages, les frontières sont autant de dimensions cachées de relations sociales codifiées et de cultures [18, 19].

Les espaces de travail sont donc parfois recadrés par le bâtiment performant, en fonction de logiques énergétiques et non pas en fonction des logiques que les occupants souhaiteraient voir reconnues : activité individuelle et collective, sociabilité, appropriation, culture. Et ce recadrage rencontre actuellement des logiques managériales de gestion des coûts, des surfaces et des salariés.

\subsection{Qu'est-ce qu'être citoyen en entreprise ?}

Un discours institutionnel appelle les individus à devenir citoyens et acteurs. Le consommateur-citoyen est par exemple une figure contemporaine des politiques publiques [14]. Mais l'individu doit être, de plus en plus, citoyen sur son lieu de travail également. ${ }^{\mathrm{e}}$

Cependant, les différents acteurs portent plus ou moins cette figure. Les concepteurs de bâtiments performants pensent l'usager comme devant s'adapter au bâtiment, nous l'avons vu (figure passive). Par contre, les directions se trouvent fortement incitées à réaliser des actions de sensibilisation, à « activer» les occupants, sans toujours maîtriser les messages, les moyens d'action ni le calendrier. Se développe alors une injonction contradictoire : enjointes de développer un discours sur la responsabilité des occupants, elles doivent également les empêcher de toucher aux systèmes.

Cette figure de l'occupant citoyen touche à la question des «valeurs » et des «pratiques », des « conduites » individuelles et sociales, et porte une tension entre situation de travail et domaine privé ; entre prescription et libre-arbitre. Nous avons vu que l'énergie est un objet hybride, pas directement outil de travail mais moyen de réaliser les activités de travail. La frontière entre ce qu'il est légitime ou non de prescrire autour de l'énergie reste souvent floue, du côté des occupants

\footnotetext{
e «Avant même l'engagement d'opérations de travaux, la modification des comportements et une meilleure gestion des locaux par les utilisateurs peuvent entraîner une baisse significative (jusqu'à $20 \%$ ) de la consommation d'énergie. Parce qu'il occupe un bureau ou habite un logement, chacun peut, à son niveau, agir sur la consommation d'énergie du bâtiment. », Rapport d'évaluation du Grenelle de l'Environnement, octobre 2010. http://lesrapports.ladocumentationfrancaise.fr/BRP/104000587/ $0000 . \mathrm{pdf}$
} 
comme du côté des directions, qui restent assez « mal à l'aise » sur le thème. En effet, la logique citoyenne pousse vers une certaine moralisation des comportements, c'est-à-dire des partitions en bonnes ou mauvaises conduites. Si l'on retrouve une certaine hésitation des directions à développer un discours moralisateur, les dispositifs sociotechniques sont considérés par les occupants comme moralisateurs, en particulier sur la définition du travail. Ainsi, ce qui était censé être invisible (les systèmes) devient un cadrage de pratiques, voire une sorte de moralisation des comportements : les systèmes définissent ce qu'est le travail (du mouvement, sinon les lumières s'éteignent), ce qu'est l'heure de pause (à heures fixes - par exemple midi et $18 \mathrm{~h}$-, les lumières s'éteignent), ce que sont les espaces à investir ou pas (les couloirs considérés comme espace "non productifs » ne sont plus chauffés dans certains bâtiments), etc.

Notons que la logique citoyenne est d'autant plus poussée en entreprise que les acteurs économiques pensent que les occupants de bâtiments tertiaires n'agissent pas au nom de la logique financière, qui serait la logique prépondérante au domicile. Les études sociologiques montrent que cette assertion est doublement à nuancer : au domicile, la logique financière est certes forte, mais ce n'est pas la seule et elle relève de logiques économiques « indigènes » et non pas d'une rationalité économique « savante » $[11,20]$. De plus, sur le lieu de travail, les occupants sont intéressés par les logiques financières de l'entreprise et par les justifications données aux économies d'énergie. Le manque de justification par les directions amène par exemple les salariés à penser que " [leur] employeur veut faire des économies sur [leur] dos ». Si les occupants sont intéressés, au sens d'une demande d'information, ils pourraient également être intéressés au sens d'une demande de redistribution collective des efforts réalisés en matière d'économies d'énergie, ce qui pourrait donner lieu à d'autres acceptions de la « citoyenneté ». Cela renvoie également à une autre limite de la citoyenneté pointée par les salariés : même dans des cas où la direction mène des opérations de concertation (l'Ingénierie, La Plateforme) que les salariés saluent, une partie d'entre eux estiment que leurs capacités d'action ne sont que périphériques, voire symboliques. Les «vraies » décisions seraient prises ailleurs. Cela renvoie à la question du partage des rôles et responsabilités.

\subsection{Conflit de normes et de pratiques « environnementales »}

Le troisième dispositif de tension renvoie à la définition de «l'environnement » et est lié à la distinction privé/professionnel, d'une part, et à la distinction technique/bon sens, d'autre part. En effet, les occupants de bâtiments performants sont dubitatifs, voire méfiants quant aux certifications (HQE, $\mathrm{BBC})$, critiquant soit leur principe même, soit leur application concrète.

Les occupants développent une définition de l'environnement très liée à leurs valeurs personnelles et à leurs pratiques sociales et culturelles de l'énergie :

- La notion de HQE ou de BBC est considérée comme très limitée par rapport à la notion de développement durable, terme que les occupants utilisent pour appréhender les problématiques environnementales. Ainsi, au Labo, des salariés se demandent si un bâtiment aussi luxueux que leur nouveau bâtiment peut vraiment être HQE (climatisation, équipements technologiques de pointe, etc.). Et ils critiquent l'absence d'ascenseurs, ce qui empêche l'accès au premier étage aux personnes à mobilité réduite.

- La logique technicienne, centrée sur les systèmes, est considérée comme allant à l'encontre du "bon sens », c'est-à-dire de ce qui a été appris, incorporé, dans le cadre domestique, éventuellement suite à des campagnes publiques. Ainsi, de nombreux salariés ont appris à ouvrir leurs fenêtres au domicile, depuis leur enfance ou depuis les campagnes réalisées dans le cadre de la qualité de l'air intérieur, et perçoivent de manière très négative, et dans les discours et dans leurs perceptions sensorielles (odeurs, sensations d'étouffement, etc.), le fait que leur bâtiment soit ventilé et non pas aéré naturellement (absence de fenêtres ouvrantes). 
- La logique «bâtiment » est considérée comme très compartimentée par rapport à une logique de cohérence des comportements. Ainsi, des salariés de La Plateforme (travaillant dans le sud de la France) mentionnent l'absence d'abris pour leur voiture sur le parking, les incitant à allumer la climatisation en fin de journée dans leur véhicule, qui a fortement chauffé au soleil.

- De plus, l'environnement est considéré comme une valeur, un engagement personnel, à développer « en son âme et conscience » (versus une prescription des systèmes et/ou managériale).

Tous ces écarts créent un conflit de normes et de pratiques, certaines pratiques domestiques considérées comme environnementales étant interdites ou impossibles à mettre en place sur le lieu de travail ; et certaines prescriptions de l'employeur ou du bâtiment faites au nom de l'environnement ne relevant pas de ce domaine selon les occupants. Notons que si les occupants refusent en partie la désignation comme environnementale d'une partie des prescriptions de leur entreprise, ils peuvent également être rétifs quand celle-ci va plus loin que leurs engagements personnels dans le domaine (bilan carbone, etc.). Ce qui crée la tension est un déséquilibre (dans un sens ou dans un autre) et une incohérence entre le répertoire d'actions domestique et le répertoire d'actions professionnel.

\section{Conclusion : Les problématiques des occupants : confort et répartition des responsabilités}

Au-delà des dispositifs de tension, l'analyse montre que les occupants développent des enjeux dans des domaines où l'énergie est traversante, encastrée, mais où elle n'est pas un objectif.

Tout d'abord, le confort est un critère important et complexe du rapport entre l'occupant et le bâtiment, et entre l'occupant et la direction. La définition du confort par les occupants comporte :

- des éléments matériels (l'enjeu étant de travailler dans de meilleures conditions que précédemment, le bâtiment et les systèmes doivent s'adapter à l'activité et aux occupants et doivent faciliter le travail, et non pas le contraindre),

- sociaux (l'enjeu étant d'avoir la capacité à développer de la coopération et de la sociabilité entre collègues, mais aussi des rapports hiérarchiques où le salarié est valorisé autant que le bâtiment souvent « vitrine »- peut l'être),

- et idéologiques/de construction de visions du monde (l'enjeu étant de pouvoir mettre en cohérence les représentations/opinions/pratiques personnelles et le lieu de travail, ou en tout cas d'avoir suffisamment de libre arbitre pour décider de manière discrétionnaire le type de pratiques et de valeurs à développer).

Ainsi, les notions de partage des responsabilités et de libre arbitre apparaissent en filigrane des discours :

- Les occupants peuvent avoir l'impression de ne pas être traités en adulte responsable par leur direction - qui ne leur fait pas partager les enjeux - et par le bâtiment - qui les infantilise, les moralise. Ils peuvent être soumis à des discours culpabilisants sur leurs pratiques et leurs " petits gestes », alors même qu'ils ressentent un manque d'emprise face aux systèmes et au bâtiment et face au management mis en place ;

- Ils peuvent également considérer qu'ils sont mis de côté par rapport au bâtiment qui est mis en visibilité dans une communication externe, par exemple inauguré en grande pompe, sans eux (bâtiment vitrine) ; alors même qu'il leur est demandé un engagement, une participation dans le projet « bâtiment».

Cela pose la question de la «participation » des occupants au projet «bâtiment », rejetée au moment de la conception, oubliée au moment de l'inauguration, mais imposée ensuite. 
La transition énergétique : vrais enjeux, faux départs?

\section{Références}

[1] A. Dujin, I. Moussaoui, X. Mordret et B. Maresca, Les usages de l'énergie dans les entreprises du secteur tertiaire. Des systèmes techniques aux pratiques, Paris, CREDOC, Cahier de Recherche No. 287 (2011) (http://www.credoc.fr/pdf/Rech/C287.pdf)

[2] C. Cooremans, "Strategic fit of energy efficiency (strategic and cultural dimensions of energyefficiency investments)", ECEEE Summer Study proceedings, pp. 73-82 (2007) (http://www . eceee.org/library/conference_proceedings/eceee_Summer_Studies/2007/Panel_ $1 / 1.177 /$ paper)

[3] D. Desjeux, A.-S. Boisard, La maîtrise de l'énergie pour les entreprises, une démarche sous contrainte, Observatoire de l'Énergie entreprises (2010) (http://www.observatoireenergies-entreprises.fr/wp-content/uploads/2009/12/la-maitrise-de-le28099 energie-pour-les-entreprises-une-demarche-sous-contrainte-d-desjeux1.pdf)

[4] G. Brisepierre, Pratiques de consommation d'énergie dans les bâtiments performants : consommation théorique et consommation réelle. La Cité de l'Environnement, premier bâtiment tertiaire à énergie positive, rapport ADEME/GBS/Leroy-Merlin Source (2012) (http:// gbrisepierre.fr/wp-content/uploads/2013/07/GBS-Monographie-BEPOS-Cit\%C3\%A9 -de-1Environnement-2013.pdf)

[5] S. Guy, E. Shove, A sociology of energy, buildings and the environment, Londres, Routledge (2000). ISBN: 978-0-415-18269-0

[6] I. Garabuau-Moussaoui, «Les occupants de bâtiments performants en énergie en sont-ils les usagers ? Production et usages de l' (in)visibilité du bâtiment, entre bâtiment vitrine, mythe de l'automatisme et souhait de la banalité », in J. Cihuelo, C. Grandclément et A. Jobert, Énergie, technologie, société, (titre provisoire), (à paraître)

[7] S. Thiriot, L'occupation d'un bâtiment performant tertiaire : étude des liens entre conception, maintenance et occupation, Mémoire de Master 2, Master « Techniques, Sciences, Décisions », INP Grenoble, sous la direction de Thomas Reverdy (PACTE, Grenoble) et Isabelle Moussaoui (EDF R\&D) (2013)

[8] M. Akrich, "The De-scription of Technical Objects", in W. Bijker and J. Law (ed), Shaping Technology/Building Society, Cambridge Massachusetts, The MIT Press, pp. 205-224 (2000). ISBN: 9780262521949

[9] H. Rohracher, "From Passive Consumers to Active Participants: The Diverse Roles of Users in Innovation Processes", in H. Rohracher (dir), User involvment in Innovation Processes. Strategies and Limitations from a Socio-Technical Perspective, München, Profil, pp. 9-35 (2005). ISBN: 389019-579-2

[10] H. Wilhite, "Will efficient technologies save the world? A call for new thinking on the ways that end-use technologies affect energy using practices", ECEEE Summer Study Proceedings, pp. 23-30 (2007) http://www.eceee.org/library/conference_proceedings/eceee_ Summer_Studies/2007/Panel_1/1.043/paper

[11] I. Garabuau-Moussaoui, «De la société de consommation à la société de modération. Ce que les Français disent, pensent et font en matière de maîtrise de l'énergie », Les Annales de la recherche urbaine, $n^{\circ}$ 103, pp.112-119 (2007). ISBN: 0180-930-X

[12] M.-Ch. Zélem, Politique de maîtrise de la demande d'énergie et résistances au changement. Une approche socio-anthropologique, Paris, L'Harmattan (2010). ISBN/EAN : 978-2-296-11438-8

[13] E. Pautard, Vers la sobriété électrique : politiques de maîtrise des consommations et pratiques domestiques, thèse de doctorat, sous la direction de Jean-Yves Nevers (2009) (http: //www . edf . com/fichiers/fckeditor/Commun/Innovation/theses/ThesePautard.pdf)

[14] M. Brugidou, I. Garabuau-Moussaoui, "From Energy Policies to Energy-related Practices in France: The Figure of the "Consumer Citizen" as a Normative Compromise", in S. Fudge, 


\section{SHS Web of Conferences}

M. Peters, S.M. Hoffman and W. Wehrmeyer, The Global Challenge Of Encouraging Sustainable Living. Opportunities, Barriers, Policy and Practice, Elgar Publishing (2013). ISBN: 978178100 3749

[15] A. Monjaret (dir.), L'alimentation au travail, Consommations et Sociétés, Cahiers pluridisciplinaires sur la consommation et l'interculturel, No. 2, Paris, L'Harmattan (2001). ISBN: 2-7475-2370-5

[16] S. Broadbent, L'intimité au travail. la vie privée et les communications personnelles dans l'entreprise, Éditions FYP (2011). ISBN-10: 2916571507 / ISBN-13: 978-2916571508

[17] E. Pélegrin-Genel, Des souris dans un labyrinthe. Décrypter les ruses et manipulations de nos espaces quotidiens, Paris, La Découverte (2010). ISBN: 2359250213 / EAN: 978-2359250213

[18] E.T. Hall, La dimension cachée, Paris, Seuil (1984). ISBN-10: 2020047764 / ISBN-13: 9782020047760

[19] P. Dibie, Ethnologie de la porte, Paris, Métailié (2012). ISBN: 9782864248415

[20] F. Weber, « Le calcul économique ordinaire », in Ph. Steiner et F. Vatin (dir), Traité de sociologie économique, Paris, PUF, pp. 367-407 (2009). ISBN: 978-2-13-056867 